\title{
Combined VLA-4-Targeted Radionuclide Therapy and Immunotherapy in a Mouse Model of Melanoma
}

\author{
Jaeyeon Choi ${ }^{1}$, Wissam Beaino ${ }^{2,3}$, Ronald J. Fecek ${ }^{4}$, Kellsye P.L. Fabian ${ }^{5}$, Charles M. Laymon ${ }^{2}$, Brenda F. Kurland ${ }^{6}$, \\ Walter J. Storkus ${ }^{1,4,5}$, and Carolyn J. Anderson ${ }^{1,2,7,8}$ \\ ${ }^{I}$ Department of Bioengineering, University of Pittsburgh, Pittsburgh, Pennsylvania; ${ }^{2}$ Department of Radiology, University of \\ Pittsburgh, Pittsburgh, Pennsylvania; ${ }^{3}$ VU University Medical Center, Department of Radiology and Nuclear Medicine, Amsterdam, \\ The Netherlands; ${ }^{4}$ Department of Dermatology, University of Pittsburgh, Pittsburgh, Pennsylvania; ${ }^{5}$ Department of Immunology, \\ University of Pittsburgh, Pittsburgh, Pennsylvania; ${ }^{6}$ Department of Biostatistics, University of Pittsburgh, Pittsburgh, Pennsylvania; \\ ${ }^{7}$ Department of Medicine, University of Pittsburgh, Pittsburgh, Pennsylvania; and ${ }^{8}$ Department of Pharmacology and Chemical \\ Biology, University of Pittsburgh, Pittsburgh, Pennsylvania
}

Very late antigen-4 (VLA-4; also known as integrin $\alpha_{4} \beta_{1}$ ) is expressed at high levels in aggressive and metastatic melanoma tumors and may provide an ideal target for imaging and targeted radionuclide therapy (TRT). ${ }^{177}$ Lu-DOTA-PEG 4 -LLP2A ( ${ }^{177}$ Lu-LLP2A) is a TRT that shows high affinity for VLA-4 and high uptake in B16F10 mouse melanoma tumors in vivo. Here, we report efficacy studies of ${ }^{177} \mathrm{Lu}-$ LLP2A, alone and combined with immune checkpoint inhibitors (ICls) (anti-PD-1, anti-PD-L1, and anti-CTLA-4 antibodies), in B16F10 tumor-bearing mice. Methods: Tumor cells $\left(1 \times 10^{6}\right)$ were implanted subcutaneously in C57BL/6 mice. After 8-10 d, the mice were randomized into 8 groups. ${ }^{177}$ Lu-LLP2A was injected intravenously on day 8 or 9 (single dose), and $\mathrm{ICl}$ antibodies were administered intraperitoneally in 3 doses. Tumor growth was monitored over time via calipers. Terminal deoxynucleotidyl transferase dUTP nick-end labeling (TUNEL) staining for apoptosis was performed on fixed tumors. In a separate study, Cy3-LLP2A or Cy3-scrambled LLP2A was injected in tumor-bearing mice, and tumors were collected $4 \mathrm{~h}$ after injection and then analyzed by flow cytometry and immunofluorescence microscopy using different immune cell markers. Results: TRT alone showed efficacy comparable to the dual-ICl anti-PD-1 + antiCTLA-4 or anti-PD-L1 + anti-CTLA-4, whereas TRT + ICls significantly enhanced survival. TUNEL staining showed that the highest levels of apoptosis were in the TRT $+\mathrm{ICl}$ groups. In addition to targeting tumor cells, TRT also bound immune cells in the tumor microenvironment. Flow cytometry data showed that the tumors consisted of about $77 \%$ tumor cells and fibroblasts (CD45-negative/ CD49d-positive) and about 23\% immune cells (CD45-positive/ CD49d-positive) and that immune cells expressed higher levels of VLA-4. Cy3-LLP2A and CD49d colocalized with macrophages (CD68), T cells (CD8, CD4), and B cells (CD19). Immunohistochemical analysis identified a significant colocalization of Cy3-LLP2A and CD68. Conclusion: Combination treatment with TRT + ICls targets both tumor cells and immune cells and has potential as a therapeutic agent in patients with metastatic melanoma.

\footnotetext{
Received Feb. 6, 2018; revision accepted May 31, 2018.

For correspondence or reprints contact: Carolyn J. Anderson, Department of Medicine, University of Pittsburgh, 3501 Fifth Ave., Room 10020, Pittsburgh, PA 15260.

E-mail: andersoncj@upmc.edu

Published online Jun. 29, 2018.

COPYRIGHT (C 2018 by the Society of Nuclear Medicine and Molecular Imaging.
}

Key Words: melanoma; immune checkpoint inhibitors; targeted radionuclide therapy; VLA-4; PET imaging

J Nucl Med 2018; 59:1843-1849

DOI: 10.2967/jnumed.118.209510

$\mathbf{M}$ elanoma is one of the most serious types of skin cancer and can develop from pigmented cells in the skin, eyes, and mucosal organs, causing high morbidity and mortality in patients (1). Surgical excision is usually curative for early-stage melanoma; however, aggressive melanomas may quickly metastasize to distal organs (2). Over the past decade, immunotherapy with immune checkpoint inhibitors (ICIs) (anti-CTLA-4, anti-PD-1, and antiPD-L1 antibodies) has demonstrated dramatic therapeutic efficacy in many patients with metastatic melanoma, and there are now several Food and Drug Administration-approved ICI-based drugs (3). However, the therapeutic response in patients is uneven because of the many molecular mechanisms regulating antitumor immune responses in cancer patients (4). Novel therapeutic approaches, including combination therapies $(5,6)$, are needed to improve overall survival rates. It was reported that combining anti-PD-1 + anti-CTLA-4 or anti-PD-L1 + anti-CTLA-4 dualICI therapy with external-beam irradiation improved efficacy in melanoma-bearing mice when compared with treatment with dualICI therapy alone (5). Radiation is not ideal, however, for treating patients with disseminated melanomas, as it cannot be applied to microscopic metastatic disease and can damage normal tissues or organs surrounding the tumors. Therefore, a systemically targeted radionuclide therapy (TRT) would be preferred to enhance the combined antitumor efficacy of radiation with ICI therapy.

Very late antigen-4 (VLA-4; also known as integrin $\alpha_{4} \beta_{1}$ ) plays an important role in tumor growth, angiogenesis, and metastasis by promoting adhesion and migration of cancer cells. VLA-4 is expressed in many types of cancer, including melanoma, lymphoma, and multiple myeloma $(7,8)$. VLA-4 also plays a crucial role in lymphocyte differentiation and trafficking (9) and has been a highly studied therapeutic target because of its immunomodulatory activity $(10,11)$.

Very high affinity and specificity for activated VLA-4 has been shown by the peptidomimetic LLP2A ( $N$-[[4-[[[(2-ethylphenyl) 


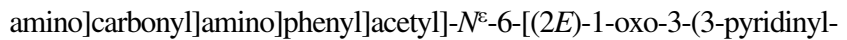
2-propenyl)]-L-lysyl-L-2-aminohexanedioyl-(1-amino-1-cyclohexane) carboxamide; Supplemental Fig. 1 [supplemental materials are available at http://jnm.snmjournals.org]) (12). LLP2A has been labeled with the $\beta$-emitter ${ }^{177} \mathrm{Lu}$ (half-life, $6.7 \mathrm{~d}$; maximum $\beta^{-}$energy, $0.49 \mathrm{MeV}$ ) and the positron emitter ${ }^{68} \mathrm{Ga}$ (half-life, $68 \mathrm{~min}$; maximum $\beta^{+}$energy, $1.92 \mathrm{MeV}$ ) for therapy and PET imaging, respectively. ${ }^{177} \mathrm{Lu}$ is an attractive radionuclide for TRT, as it has a moderate $\beta^{-}$ energy that is effective for treatment of smaller metastatic tumors. The half-life of ${ }^{68} \mathrm{Ga}$ is amenable to imaging with peptides and small molecules. We previously reported that ${ }^{68} \mathrm{Ga}$ - and ${ }^{177} \mathrm{Lu}$-labeled LLP2A have high uptake in B16F10 mouse melanoma tumors (13).

The primary objective of this study was to investigate the antitumor efficacy of combining the TRT ${ }^{177} \mathrm{Lu}$-LLP2A with ICIs in B16F10 tumor-bearing mice versus using either as a singlemodality treatment. We also identified VLA-4-expressing cell types within the tumor microenvironment (TME) and determined expression levels of VLA-4 in immune cells in the TME, with a particular interest in those known to have a strong impact on tumor progression and metastasis (14).

\section{MATERIALS AND METHODS}

\section{Reagents}

All chemicals were purchased from Sigma-Aldrich unless otherwise specified. Aqueous solutions were prepared using ultrapure water (resistivity, $18.2 \mathrm{M} \Omega \cdot \mathrm{cm}$ ). Rink amide 4-methylbenzhydrylamine resin (loading, $0.77 \mathrm{mmol} / \mathrm{g}$ ) and all fluorenylmethyloxycarbonyl-protectinggroup (Fmoc)-protected amino acids were purchased from ChemImpex International, Inc. DOTA was purchased from Chematech. Fmoc-polyethylene glycol $\left(\mathrm{PEG}_{4}\right)$ carboxylic acid was purchased from ChemPep Inc. Cy3 carboxylic acid was purchased from Lumiprobe. All flow cytometry antibodies were obtained from BD Biosciences. ${ }^{177} \mathrm{LuCl}_{3}$ was purchased from the University of Missouri Research Reactor. Anti-PD-L1 (clone, 10F.9G2), anti-PD-1 (clone, RMP1-14), and anti-CTLA-4 (clone, 9H10) antibodies were obtained from BioXCell. C57BL/6 mice were purchased from Charles River Laboratories.

\section{Instrumentation}

Analytic and semipreparative reversed-phase high-performance liquid chromatography was performed on a Waters 1525 Binary Pump with a Waters 2489 UV/Vis detector and a model 106 Bioscan radioactivity detector. LLP2A analogs were purified on a semipreparative Jupiter C18 column (Phenomenex). Radiochemistry reaction progress and purity were monitored on a Jupiter C18 column. Radioactive samples were counted using either an automated Packard Cobra II $\gamma$-counter or a PerkinElmer 2470 Wizard $^{2}$ automatic $\gamma$-counter. PET/CT data were acquired on an Inveon Preclinical Imaging Station (Siemens Medical Solutions). Microscopy images were acquired by immunofluorescence (Zeiss Microscopy) and analyzed with ZEN 2009 software (Zeiss Microscopy). Tumor cells and immune cells were analyzed with an LSRII flow cytometer (BD Biosciences) and FlowJo software (BD Biosciences).

\section{Peptide Synthesis and Radiolabeling Studies}

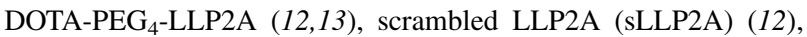
and Cy3-PEG 4 -conjugated LLP2A and sLLP2A (15) were synthesized as previously described. ${ }^{177} \mathrm{Lu}$ - and ${ }^{68} \mathrm{Ga}$-labeled $\mathrm{DOTA}^{-\mathrm{PEG}_{4}-}$ LLP2A $\left({ }^{177} \mathrm{Lu}-\mathrm{LLP} 2 \mathrm{~A} ;{ }^{68} \mathrm{Ga}\right.$-LLP2A) were prepared in greater than $99 \%$ purity as previously described (13).

\section{In Vivo TRT}

All animal studies were conducted under protocols approved by the University of Pittsburgh Institutional Animal Care and Use Committee. B16F10 tumor cells $\left(1 \times 10^{6}\right)$ were injected subcutaneously in the left flank of female and male C57BL/6 mice (4-6 wk old). Therapy studies commenced when tumors were palpable (8-10 d after tumor injection). Mice receiving single- or dual-ICI therapy without TRT were injected intraperitoneally with anti-CTLA-4, anti-PD-L1, or anti-PD-1 antibodies $(200 \mu \mathrm{g})$ on days 9,12 , and 15 after tumor cell injection. Mice receiving TRT were injected intravenously with $30 \mathrm{MBq}$ of ${ }^{177} \mathrm{Lu}-$ LLP2A either alone or before ICI therapy.

The dose scheduling for all groups of mice is summarized in Figure 1. Body weight and tumor volume were measured every $2 \mathrm{~d}$ until 13 $\mathrm{d}$ after the start of therapy. After day 13, the tumors were measured daily. The tumor volumes were based on caliper measurements and estimated using the equation [volume $=(\pi \div 6) \times \mathrm{L} \times \mathrm{W} \times \mathrm{H}$ ], where $\mathrm{L}$ is the longest axis, $\mathrm{W}$ is the axis perpendicular to $\mathrm{L}$, and $\mathrm{H}$ is the axis perpendicular to the $\mathrm{L}$ and $\mathrm{W}$ planes. The mice were sacrificed when the tumor ulcerated or reached a volume of $1.5 \mathrm{~cm}^{3}$ in the longest axis. The mice showed no signs of pain, distress, or morbidity (scruffy coat, diarrhea, lethargy, 20\% weight loss).

\section{PET/CT Imaging}

Tumor-bearing mice were injected intravenously with ${ }^{68} \mathrm{Ga}$-LLP2A $(200 \mu \mathrm{Ci} ; 7.4 \mathrm{MBq})$ and anesthetized with $2 \%$ isoflurane, and static $\mathrm{PET} / \mathrm{CT}$ images were obtained at 1 and $2 \mathrm{~h}$ after injection. The PET and CT images were coregistered and analyzed with Inveon Research Workstation software as previously described (13).

\section{Immunofluorescence Microscopy}

Tumor-bearing mice were injected intravenously with either Cy3LLP2A or Cy3-sLLP2A (50 $\mu \mathrm{g} ; n=2$ for each group). At $4 \mathrm{~h}$ after injection, the mice were euthanized, and the tumors were collected and frozen. Sections $(6 \mu \mathrm{m})$ were obtained, and nonspecific blocking was performed ( $2 \%$ bovine serum albumin). Sections were then stained with antimouse CD49d-FITC (clone, R12), antimouse CD68-APC (clone, FA-11), and 4',6-diamino-2-phenylindole (DAPI). After washing in phosphate-buffered saline, coverslips were mounted using Prolong Diamond Antifade Mountant (Thermo Fisher). The slides were imaged with a Zeiss Apotome system equipped with a Zeiss plan Apochromat $\times 63$ high-performance objective with an oil immersion lens (numeric aperture, 1.4-0.6).

\section{Terminal Deoxynucleotidyl Transferase dUTP Nick-End Labeling (TUNEL) Staining}

At the time of euthanasia after therapy, tumors from 6 treatment groups (1. Control [nontreated]; 2. ${ }^{177}$ Lu-LLP2A [TRT alone]; 3. anti-PD-1 and

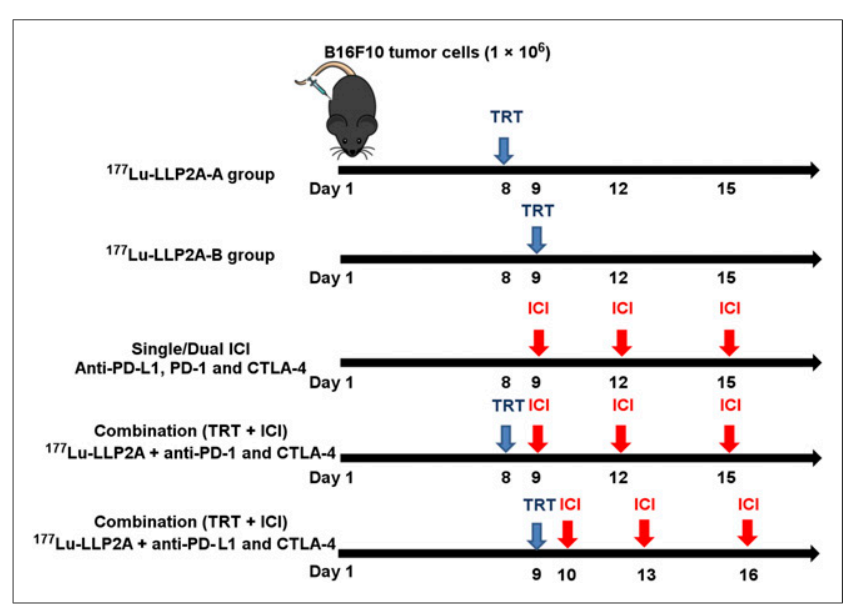

FIGURE 1. TRT and ICl schedule. ${ }^{68} \mathrm{Ga}-\mathrm{LLP} 2 \mathrm{~A}$ imaging of TRT + antiPD-L1 + anti-CTLA-4 group was performed 22 d after tumor cell implantation. 
anti-CTLA-4 [ICI dual]; 4. anti-PD-L1 and anti-CTLA-4 [ICI dual]; 5. ${ }^{177}$ Lu-LLP2A + anti-PD-L1 and anti-CTLA-4 [combination TRT + ICI]; and $6 .{ }^{177} \mathrm{Lu}-\mathrm{LLP} 2 \mathrm{~A}+$ anti-PD-1 and anti-CTLA-4 [combination TRT + ICI]) were excised and fixed in $4 \%$ formaldehyde (in phosphatebuffered saline) for $30 \mathrm{~min}$ at room temperature, followed by embedding in paraffin. The paraffin blocks were sectioned $(5 \mu \mathrm{m})$, and apoptotic cell death was assessed via TUNEL staining (Sigma Aldrich) on the Apotome system. Nuclei were counterstained with DAPI. For analysis, the DAPIcounterstained cells (representing total cells) for an entire $\times 10$ field of view were counted using ZEN software, followed by counting of TUNELpositive cells. The percentage of apoptotic cells in the field was based on the ratio of TUNEL-positive and DAPI-counterstained cells. Scores were averaged for 3 fields of view for control, TRT alone, dual ICI (anti-PD-1 + anti-CTLA-4 or anti-PD-L1 + anti-CTLA-4), and TRT + ICI (TRT + anti-PD-1 + anti-CTLA-4 or TRT + anti-PD-L1 + anti-CTLA-4).

\section{Flow Cytometry}

Cy3-LLP2A $(50 \mu \mathrm{g})$ or Cy3-sLLP2A $(50 \mu \mathrm{g})$ was injected intravenously into tumor-bearing mice ( $n=2$ per group). At $4 \mathrm{~h}$ after injection, the mice were euthanized, and the tumors were collected, incubated with a mixture of collagenase D $(2 \mathrm{mg} / \mathrm{mL})$ and DNase-I (1/ 100 , stock solution at $1 \mathrm{mg} / \mathrm{mL}$ ) in RPMI $1640 /$ fetal bovine serum (2\%) for $45 \mathrm{~min}$ at $37^{\circ} \mathrm{C}$, and passed through a mesh filter $(70 \mu \mathrm{m})$ to create a single-cell suspension. Red blood cells were lysed with ammonium-chloride-potassium buffer (Thermo Fisher), and nucleated cells were washed with phosphate-buffered saline. The cells were then labeled with a mixture of the following antimouse fluorescein isothiocyanate (FITC)-conjugated antibodies for $30 \mathrm{~min}$ at room temperature: CD49d FITC (clone, R12), CD45 PE (clone, 104), CD4 PE (clone, RM4-5), CD8 FITC (clone, 53-6.7), CD11c FITC (clone, 6D5), CD68 FITC (clone, FA-11), FoxP3 FITC, PD-1 FITC (clone, CD279), PD-L1 FITC (clone, 10F.9G2), and NK FITC (clone, 29A1.4). The cells were analyzed by flow cytometry using LSRFortessa (BD Biociences). Gating first was done on the forward-light-scatter/side-scatter characteristics of the cells and then was based on VLA-4 expression. For all Cy3-LLP2Apositive live cells, tumor cells were identified as CD45-negative/CD49dpositive. Immune cells were identified as CD45-positive/CD49d-positive and were then stained with the antibodies listed above.

\section{Human Dosimetry}

${ }^{177} \mathrm{Lu}-\mathrm{LLP} 2 \mathrm{~A}(5-6 \mathrm{MBq} / \mathrm{nmol})$ was injected intravenously into ICR non-tumor-bearing mice, and biodistribution was measured from $1 \mathrm{~h}$ to $7 \mathrm{~d}$ after injection ( $n=5$ per group). Time-activity curves weighted by organ masses (to facilitate scaling to human values (16)) per the injected dose for 20 organs (Table 1) were determined and integrated to determine organ residence times. Mouse organ residence times were rescaled to human values (16) and then used as input data to OLINDA/ EXM, version 1.0, to estimate male and female human absorbed radiation doses. Fat and blood are not source organs in OLINDA/EXM, and surveyed activity for these components was treated as "remainder," assumed to be distributed throughout the body. It was assumed that for each time point, $95 \%$ of the actual activity present was accounted for in the organ assay; the remaining 5\% was added to the remainder. Conversion of mouse to human remainder assumed that the ratio of mouse remainder to activity in OLINDA source organs was constant between humans and mice. Although activity in bone as a whole was surveyed, the distribution of activity between bone marrow, trabecular bone, and cortical bone was not determined. In the conversion to human values, it was assumed that activity was distributed among these components on the basis of their relative masses in humans.

\section{Statistics}

SAS/STAT software, version 9.4 (SAS Institute, Inc.), and Prism software, version 7 (GraphPad), were used for statistical analysis.
TABLE 1

\section{Estimated Human Dosimetry of ${ }^{177}$ Lu-LLP2A Calculated Using OLINDA/EXM Program}

\begin{tabular}{lcc}
\hline & \multicolumn{2}{c}{ Dose $(\mathrm{Sv} / \mathrm{MBq})$} \\
\cline { 2 - 3 } \multicolumn{1}{c}{ Organ } & Male & Female \\
\hline Adrenals & $1.53 \mathrm{E}-02$ & $1.96 \mathrm{E}-02$ \\
\hline Brain & $1.94 \mathrm{E}-03$ & $2.45 \mathrm{E}-03$ \\
\hline Breasts & $2.61 \mathrm{E}-03$ & $3.11 \mathrm{E}-03$ \\
\hline Gallbladder wall & $1.03 \mathrm{E}-02$ & $1.16 \mathrm{E}-02$ \\
\hline Lower large intestine wall & $1.31 \mathrm{E}-02$ & $1.68 \mathrm{E}-02$ \\
\hline Small intestine & $2.25 \mathrm{E}-02$ & $2.88 \mathrm{E}-02$ \\
\hline Stomach wall & $5.93 \mathrm{E}-03$ & $7.25 \mathrm{E}-03$ \\
\hline Upper large intestine wall & $1.53 \mathrm{E}-02$ & $1.94 \mathrm{E}-02$ \\
\hline Heart wall & $1.09 \mathrm{E}-02$ & $1.39 \mathrm{E}-02$ \\
\hline Kidneys & $3.69 \mathrm{E}-02$ & $4.76 \mathrm{E}-02$ \\
\hline Liver & $4.52 \mathrm{E}-02$ & $5.82 \mathrm{E}-02$ \\
\hline Lungs & $2.67 \mathrm{E}-02$ & $3.45 \mathrm{E}-02$ \\
\hline Muscle & $2.38 \mathrm{E}-02$ & $3.06 \mathrm{E}-02$ \\
\hline Ovaries & $3.99 \mathrm{E}-03$ & $4.69 \mathrm{E}-03$ \\
\hline Pancreas & $2.52 \mathrm{E}-02$ & $3.23 \mathrm{E}-02$ \\
\hline Red marrow & $8.49 \mathrm{E}-02$ & $8.34 \mathrm{E}-02$ \\
\hline Osteogenic cells & $3.14 \mathrm{E}-01$ & $4.46 \mathrm{E}-01$ \\
\hline Skin & $2.67 \mathrm{E}-03$ & $3.15 \mathrm{E}-03$ \\
\hline Spleen & $3.15 \mathrm{E}-01$ & $4.08 \mathrm{E}-01$ \\
\hline Testes & $2.91 \mathrm{E}-03$ & \\
\hline Thymus & $6.01 \mathrm{E}-02$ & $7.74 \mathrm{E}-02$ \\
\hline Thyroid & $1.38 \mathrm{E}-02$ & $1.74 \mathrm{E}-02$ \\
\hline Urinary bladder wall & $7.15 \mathrm{E}-03$ & $8.86 \mathrm{E}-03$ \\
\hline Uterus & $3.77 \mathrm{E}-03$ & $4.38 \mathrm{E}-03$ \\
\hline Total body & $2.27 \mathrm{E}-02$ & $2.75 \mathrm{E}-02$ \\
\hline Effective dose equivalent & $5.36 \mathrm{E}-02$ & $6.71 \mathrm{E}-02$ \\
\hline Effective dose & $3.15 \mathrm{E}-02$ & $3.02 \mathrm{E}-02$ \\
\hline
\end{tabular}

Average survival from the first day of treatment (day after palpation for control groups) was compared between treatment groups using a linear mixed-effects regression model with $\log$ (survival days) as outcome, days from injection to palpation as a covariate, treatment group as the covariate of interest, and random experiment effects. Multiple comparisons were adjusted using the Dunnett test, separately for comparison of each group to the control group, to the TRT + antiPD-1 + anti-CTLA-4 group, and to the TRT + anti-PD-L1 + antiCTLA-4 group. Biodistribution was performed using the unpaired 2-tailed $t$ test. Two-sided $P$ values $(\leq 0.05)$ were considered significant.

\section{RESULTS}

\section{Radiolabeling Chemistry}

The radiochemical yields of ${ }^{177} \mathrm{Lu}-\mathrm{LLP} 2 \mathrm{~A}$ and ${ }^{68} \mathrm{Ga}-\mathrm{LLP} 2 \mathrm{~A}$ were $99 \%$ and showed greater than $98 \%$ stability in phosphatebuffered saline at $37^{\circ} \mathrm{C}$ for $6 \mathrm{~h}$.

\section{Therapeutic Efficacy}

The TRT (30 MBq), either alone or in combination with ICIs, was administered to tumor-bearing mice in 4 series of experiments 


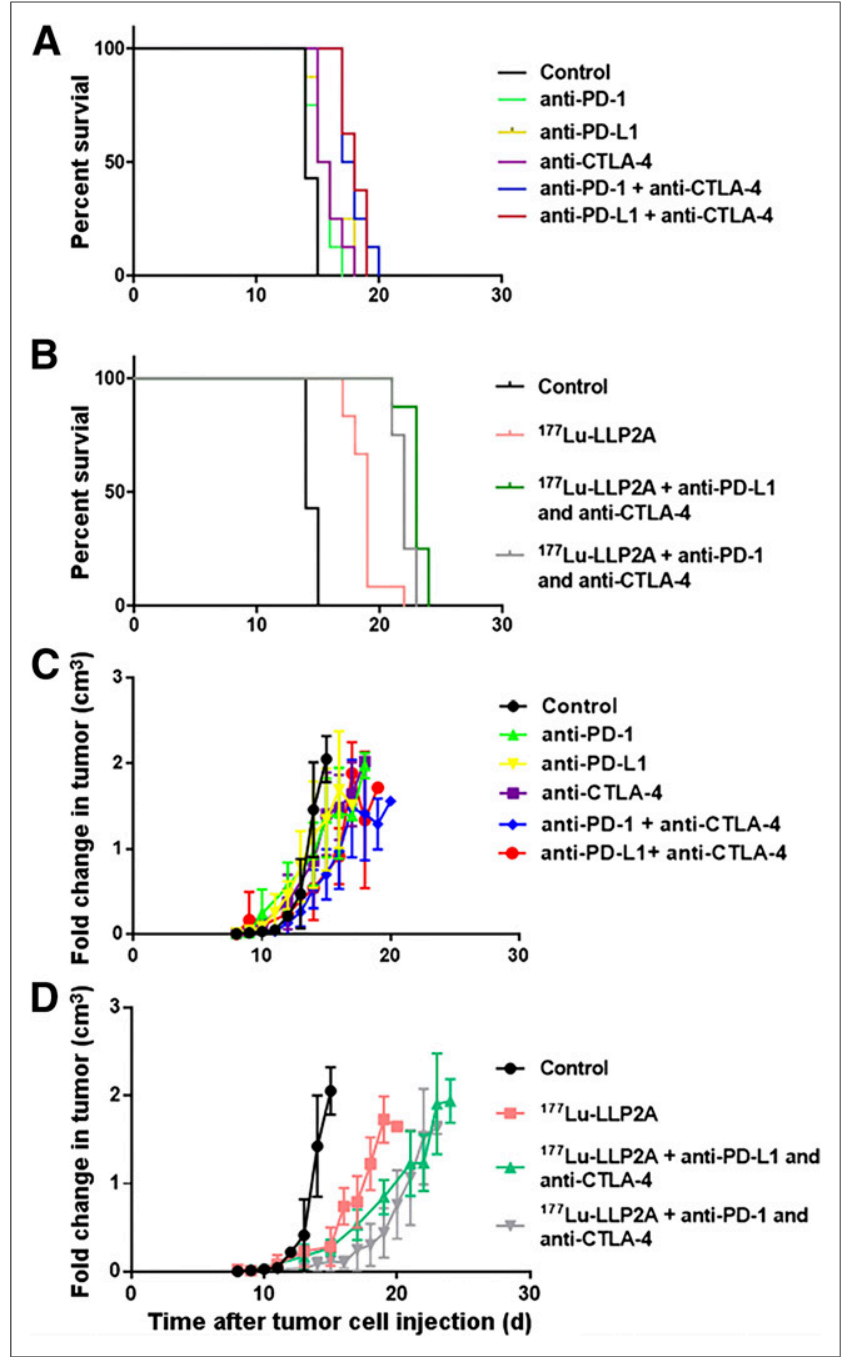

FIGURE 2. (A) Survival curves for control, single-ICl, and dual-ICl groups. (B) Survival after TRT alone and TRT + ICl. (C) Fold change in tumor size for control, single-ICl, and dual-ICl groups. (D) Fold change in tumor size for control, TRT alone, and TRT + ICl groups.

(Fig. 1). The mice were monitored for tumor size (Fig. 2) and for overt toxicity (scruffy coat, diarrhea, lethargy). All sacrifice times were determined by tumor size or ulceration rather than morbidity criteria.

The 3 single-agent ICI groups (anti-CTLA-4, anti-PD-1, and anti-PD-L1) did not show improved survival compared with the control group (Fig. 2; adjusted $P>0.9$ ). Combination TRT + ICI using TRT + anti-PD-1 + anti-CTLA-4 and using TRT + antiPD-L1 + anti-CTLA-4 had a median survival time of 22 and $23 \mathrm{~d}$, respectively, from tumor cell injection (Table 2). Survival from combination TRT + ICI was superior to that from other treatments (adjusted $P \leq 0.002$ ), except for dual-ICI (anti-PD-1 + antiCTLA-4 or anti-PD-L1 + anti-CTLA-4) versus TRT + ICI using TRT + anti-PD-1 + anti-CTLA-4 (adjusted $P=0.29$ ). Those 2 treatment groups were part of the same experiment, and the animals assigned to dual-ICI had inherently more indolent tumors, with $9 \mathrm{~d}$ from injection to palpation compared with 7 for the animals receiving TRT + ICI. ${ }^{68} \mathrm{Ga}-\mathrm{LLP} 2 \mathrm{~A}$ imaging $(2 \mathrm{~h}$ after injection) of tumor-bearing mice $6 \mathrm{~d}$ after dual-ICI using TRT + anti-PD-L1 + anti-CTLA-4 showed high tumor uptake (Fig. 3).

\section{Immune Cell Targeting by Immunohistochemistry Using Cy3-LLP2A}

To determine the immune cell types targeted by ${ }^{177} \mathrm{Lu}-\mathrm{LLP} 2 \mathrm{~A}$, 2 mice were injected in vivo with Cy3-LLP2A, and 2 were injected in vivo with Cy3-sLLP2A as a negative control (Supplemental Fig. 1). Frozen sections of the tumors isolated from these mice were stained with DAPI and showed antibodies reactive against CD68 and CD49d. The CD68 pattern of staining correlated well with that of Cy3-LLP2A and CD49d (Fig. 4). Macrophages are known to express VLA-4 on their cell surface, and these cells are a likely target of ${ }^{177}$ Lu-LLP2A. Notably, Cy3-sLLP2A exhibited negligible binding to immune cells in the TME of melanomas (Supplemental Figs. 2 and 3).

TUNEL staining was performed on tumors collected from euthanized mice. Tumors after TRT alone, ICI alone, or TRT + ICI showed greater staining than control tumors $(P<0.001$ for each, with Dunnett adjustment). The percentage of apoptotic cells in the control tumors was about $6 \% \pm 4 \%$, compared with $36 \%-$ $44 \%$ after TRT alone or ICI alone $( \pm 12 \%-14 \%)$ and $81 \%$ after TRT + anti-PD-L1 + anti-CTLA-4 $( \pm 4 \%)$ and TRT + anti-PD-1 + anti-CTLA-4 ( $\pm 8 \%$ ) (Fig. 5).

\section{Immune Cell Targeting by Flow Cytometry Using Cy3-LLP2A}

Flow cytometry was used to identify cells coexpressing Cy3LLP2A and CD49d, as well as alternate immune cell subpopulation-specific markers in the TME (Supplemental Fig. 4). Cells that stained positively for Cy3-LLP2A $(60.1 \%$ sorted by live cells in the TME) comprised 23\% immune cells (CD45-positive/CD49dpositive) and $75 \%$ tumor cells or fibroblasts (CD45-negative/ CD49d-positive) (Fig. 6A). The largest populations of Cy3LLP2A-positive cells costained for expression of PD-1 (40\%), CD68 (35\%), and PD-L1 (35\%) (Fig. 6B). However, B cells

TABLE 2

Median Survival After Single-ICI, Dual-ICI, TRT, or TRT + Dual-ICI

\begin{tabular}{|c|c|c|c|c|c|c|c|c|}
\hline \multirow[b]{2}{*}{ Control } & \multicolumn{3}{|c|}{ Single-ICI } & \multicolumn{2}{|c|}{ Dual-ICI } & \multirow[b]{2}{*}{$\begin{array}{l}\text { TRT }\left({ }^{177} \mathrm{Lu}-\right. \\
\text { LLP2A) }\end{array}$} & \multicolumn{2}{|c|}{ TRT + dual-ICl } \\
\hline & $\begin{array}{c}\text { Anti- } \\
\text { CTLA-4 }\end{array}$ & $\begin{array}{l}\text { Anti- } \\
\text { PD-L1 }\end{array}$ & $\begin{array}{l}\text { Anti- } \\
\text { PD-1 }\end{array}$ & $\begin{array}{c}\text { Anti-PD-L1 + } \\
\text { anti-CTLA-4 }\end{array}$ & $\begin{array}{l}\text { Anti-PD-1 + } \\
\text { anti-CTLA-4 }\end{array}$ & & $\begin{array}{c}\text { TRT + } \\
\text { anti-CTLA-4 + } \\
\text { anti-PD-L1 }\end{array}$ & $\begin{array}{c}\text { TRT + } \\
\text { anti-CTLA-4 + } \\
\text { anti-PD-1 }\end{array}$ \\
\hline $14 d$ & $15.5 d$ & $15.5 d$ & $15.5 d$ & $18 d$ & $17.5 \mathrm{~d}$ & $19 \mathrm{~d}$ & $23 d$ & $22 d$ \\
\hline
\end{tabular}

All treatment groups were significantly better than control $(P<0.005)$. No ICl groups significantly differed except anti-PD-L1 vs. antiPD-L1 + anti-CTLA-4 $(P=0.0082)$. TRT + dual-ICl was significantly better than TRT alone $(P<0.001)$ or dual-ICl $(P<0.0005)$. 


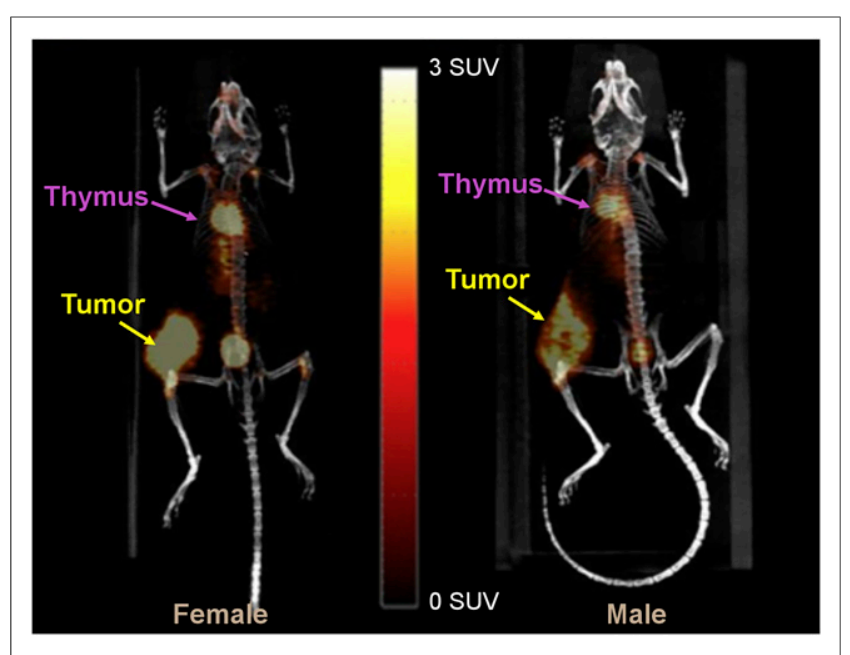

FIGURE 3. ${ }^{68} \mathrm{Ga}$-LLP2A images of mice $2 \mathrm{~h}$ after tracer injection, 6 d after treatment with TRT + anti-PD-L1 + anti-CTLA-4, and $22 \mathrm{~d}$ after tumor cell implantation. Tumor uptake is strong in left thigh, with $\mathrm{SUV}_{\text {mean }}$ of $2.3 \pm 0.5$ for female mouse and $2.0 \pm 0.3$ for male mouse.

(CD19), macrophages (CD68), and T cells (CD8 and CD4) stained most strongly with Cy3-LLP2A, suggesting that these cell types have the highest levels of VLA-4 expressed on a per-cell basis. Only $1.6 \%$ of the isotype control agent, Cy3-sLLP2A, was taken up in tumor cells in vivo $(n=12)$, compared with $58 \%$ of Cy3LLP2A $(n=12)$ (Supplemental Fig. 2).

\section{Equivalent Radiation Dose to Humans Estimated from Mouse Biodistribution}

The estimated equivalent doses of radiation from ${ }^{177} \mathrm{Lu}-\mathrm{LLP} 2 \mathrm{~A}$ to normal organs in humans were calculated on the basis of the normal biodistribution in mice (Table 1). The human models provided by OLINDA/EXM are hermaphroditic in that the male model contains female organs. These data are similar to the estimated human dosimetry of ${ }^{177} \mathrm{Lu}$-DOTATATE based on rat biodistribution (17). The equivalent dose to the spleen was higher for ${ }^{177} \mathrm{Lu}-\mathrm{LLP} 2 \mathrm{~A}$ (female, $0.41 \mathrm{mSv} / \mathrm{MBq}$; male, $0.32 \mathrm{mSv} / \mathrm{MBq}$ ) than for ${ }^{177} \mathrm{Lu}$-DOTATATE $(0.02 \mathrm{mSv} / \mathrm{MBq})$. However, the equivalent doses to the red marrow (female, $0.083 \mathrm{mSv} / \mathrm{MBq}$; male, $0.085 \mathrm{mSv} / \mathrm{MBq}$ ), adrenals (female, $0.020 \mathrm{mSv} / \mathrm{MBq}$; male, 0.015 $\mathrm{mSv} / \mathrm{MBq}$ ), and kidneys (female, $0.048 \mathrm{mSv} / \mathrm{MBq}$; male, 0.037 $\mathrm{mSv} / \mathrm{MBq}$ ) were lower for ${ }^{177} \mathrm{Lu}-\mathrm{LLP} 2 \mathrm{~A}$ than for ${ }^{177} \mathrm{Lu}-\mathrm{DOTA}-$ TATE (red marrow, $0.097 \mathrm{mSv} / \mathrm{MBq}$; adrenals, $1.4 \mathrm{mSv} / \mathrm{MBq}$; kidneys, $0.68 \mathrm{mSv} / \mathrm{MBq}$ ). However, the red marrow dose determined in the present study was an estimate.

\section{DISCUSSION}

Metastatic melanoma is challenging to treat with standard chemotherapy and radiation therapy (18). Recently, dual-ICI therapy (anti-PD-1 + anti-CTLA-4 or anti-PD-L1 + anti-CTLA-4) has exhibited enhanced efficacy in patients with metastatic melanoma compared with single-ICI therapy $(3,19)$. However, the responses to these ICIs remain uneven, and only a subset of patients receives durable clinical benefit from ICIs $(20,21)$. To overcome these limitations, combination protocols, such as ICIs + radiation, have emerged as second-line or co-first-line treatment option (5). Combining PD-1 or PD-L1 blockade with radiation was shown to yield improved treatment benefit versus radiation alone (22-24), putatively by promoting the expansion and recruitment of tumorreactive $T$ cells to tumor sites, thereby facilitating antitumor responses in vivo $(25,26)$. Although the mechanisms have not been fully elucidated, studies have shown that surface PD-L1 expression on some cancers is upregulated after a single high dose of radiation (23) or after lower doses of fractionated radiation $(22,27)$. Here, we investigated combinations of TRT + ICIs against melanoma in mice to determine whether survival benefits might be equivalent to or exceed those for radiation + ICIs.

The B16F10 melanoma model is highly resistant to ICI monotherapy; however, therapeutic synergy was observed in this model when a single 20-Gy dose of radiation was combined with ICIs (5). Our current data do not show as dramatic a benefit for TRT + ICIs, likely because the number of tumor cells implanted previously (5) was 20 -fold less than currently $\left(5 \times 10^{4}\right.$ vs. $1 \times 10^{6}$ cells). Additionally, radiation therapy was performed on day 8 in the previous study, when there most likely was a nonpalpable tumor, whereas here we started therapy after a tumor nodule had formed. The survival benefit of 3 or more days for TRT + ICIs compared with other therapies (Table 2) is modest; however, it reflects the impact of only single doses of each therapy. Additionally, the levels of apoptosis between TRT alone and TRT + ICIs is consistent with improved survival (Fig. 5).

Although radiation is often used to treat a range of localized tumors, it is not applicable for the treatment of disseminated, micrometastatic disease that cannot be visualized by standard radiologic techniques. TRT may represent an alternative systemic treatment for metastatic disease (28-30). Miao et al. investigated a ${ }^{177} \mathrm{Lu}$-DOTA-labeled $\alpha$-melanocyte-stimulating hormone peptide in B16/F1 tumor-bearing mice (31). With a single dose of 37 $\mathrm{MBq}$, they saw only modest improvement in survival (mean of $16.2 \mathrm{~d}$, vs. $13.3 \mathrm{~d}$ for the control). We hypothesized that when TRT targeting VLA-4-positive melanoma and immune cells within the

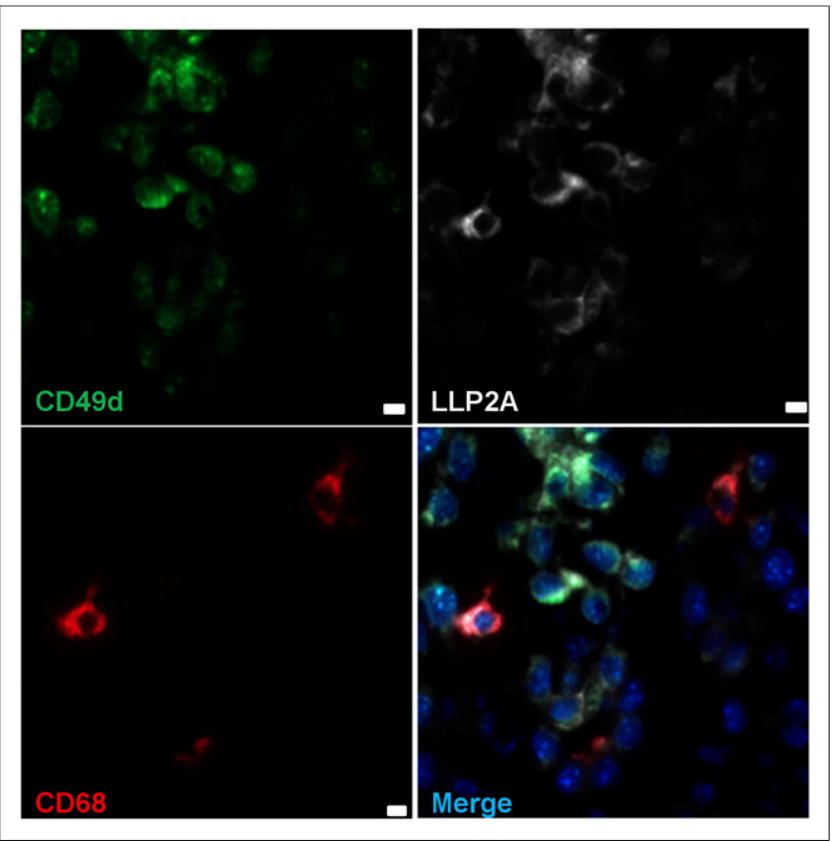

FIGURE 4. Immunofluorescence microscopy images of tumor after injection of Cy3-LLP2A in vivo (x64). Merged staining shows colocalization of Cy3-LLP2A with CD49d ( $a_{4}$ integrin subunit) and macrophages (CD68). 


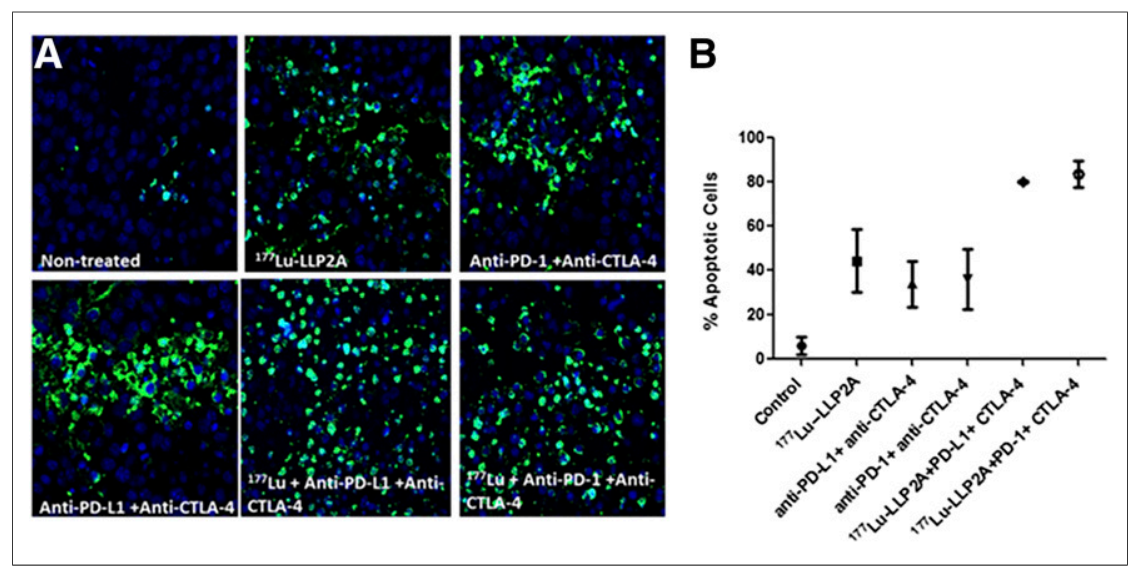

FIGURE 5. (A) Immunofluorescence microscopy images showing apoptosis (TUNEL staining) in control and all treated groups $(\times 40)$. TUNEL staining confirmed significant increase in apoptosis after treatment. (B) Percentages of apoptotic cells in treatment groups.

TME was combined with ICI, efficacy against B16 melanomas in mice would improve. In this regard, the presence of activated VLA-4 on melanoma cells correlates strongly with an aggressive, metastatic phenotype (32), as does expression of the VLA-4 integrin with melanoma progression or metastasis (32-35). Furthermore, ${ }^{177} \mathrm{Lu}-\mathrm{LLP} 2 \mathrm{~A}$, a high-affinity peptidomimetic ligand, displays selective targeting in mouse models of melanoma (13), and therapeutic efficacy is enhanced when this agent is combined with ICI therapy.

On the basis of flow cytometry data, the B16F10 tumors consisted of $75 \%$ tumor cells and fibroblasts (CD45-negative/ CD49d-positive) and 23\% immune cells (CD45-positive/CD49dpositive), and the immune cells showed higher levels of VLA-4 expression in vivo (Fig. 6). These experiments support the high avidity and specificity of LLP2A within the TME, targeting macrophages, T cells, and B cells. Although the percentage of B cells in the tumor is lower than that of $\mathrm{T}$ cells and macrophages, $\mathrm{B}$ cells have a high binding capacity for Cy3-LLP2A based on flow cytometry data. Immunofluorescence microscopy data from tumors in mice injected in vivo with Cy3-LLP2A suggest that CD68-positive cells are most commonly CD49d-positive ( $\alpha_{4}$ integrin subunit) and bind Cy3-LLP2A (Fig. 6A). The high numbers of tissue-associated macrophages correlate with strong Cy3-LLP2A staining, suggesting colocalization in macrophages, consistent with results reported for tuberculosis granulomas in a macaque model (15).

The immune response appears critical in protecting the host against the development, progression, and metastasis of tumors and is responsible for immunoediting and immune surveillance (36). Studies have also shown that certain immune cells in the TME can promote tumor progression and metastasis, suggesting dual roles for immune cells in the TME $(37,38)$. CD8positive $\mathrm{T}$ cells and natural killer cells are both well appreciated for their abilities to prevent or limit cancer metastasis $(39,40)$. Conversely, regulatory (VLA-4-positive) immune cell populations may facilitate tumor metastasis through their immunosuppressive and proangiogenic activities $(14,41)$. Indeed, VLA-4-positive myeloid cells have been reported to play a crucial role in mediating protumorigenic signals in nearly every aspect of cancer progression (42). In this regard, our data advance the field by providing important information on the types of VLA-4-positive immune cells found in the progressive TME, as well as on the relative abundance of VLA- 4 expression among the various types of immune cell infiltrates.

VLA-4-targeted TRT to immune-cell-rich tissues (spleen, thymus, bone marrow) elicits concern about a collateral negative impact on the targeting of $\mathrm{T}$ cells. We moved forward with these studies despite this issue, as immune cells will regenerate, and in cases of extreme tumor burden in metastatic melanoma, damage to organs such as the spleen can be tolerated to save the patient. Conversely, transient ablation of VLA-4-positive $\mathrm{T}$ cells has the positive impact of allowing for their compensatory homeostatic expansion and the development of a more effective, less exhausted antitumor immune response, such as has been reported for nonmyeloablative, lymphodepletion regimens (43). Future studies will determine the extent to which ${ }^{177} \mathrm{Lu}-\mathrm{LLP} 2 \mathrm{~A}$ damages protective versus regulatory immune cells in the TME as well as damage to normal tissues such as spleen, thymus, and bone marrow.

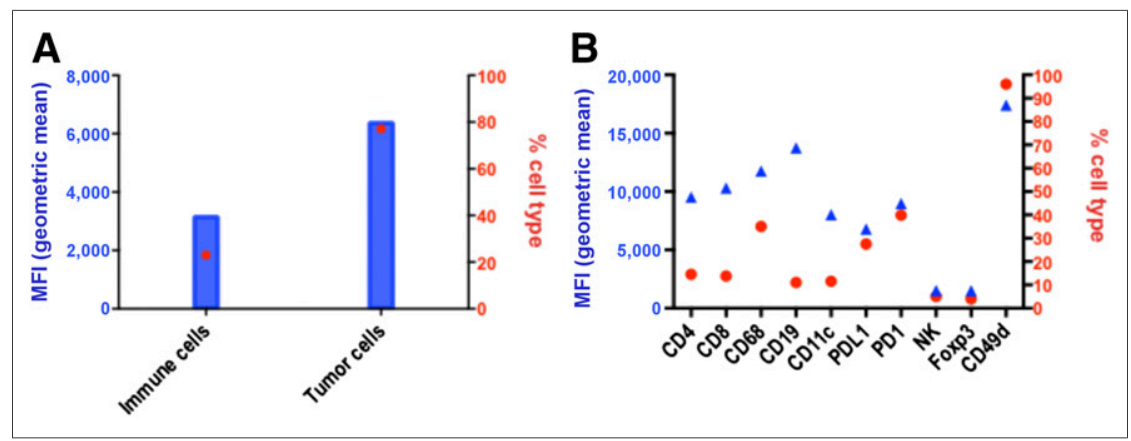

FIGURE 6. Mean florescence intensity of immune cells and tumor cells in TME (gated on live Cy3-LLP2A-positive cells). (A) Percentage of Cy3-LLP2A-positive immune cells (CD45-positive/ CD49d-positive) and tumor cells in TME was $23 \%$ and $75 \%$, respectively. Tumor cells (CD45negative/CD49d-positive) had 1.4-fold higher mean fluorescence intensity than immune cells (CD45-positive/CD49d-positive). (B) Geometric mean florescence intensity of LLP2A-positive immune cells in TME. Data show that of CD49d-positive cells, immune cells expressing CD19, CD68, PD-1, and CD8 bind most Cy3-LLP2A, indicating high levels of VLA-4.

\section{CONCLUSION}

The data presented here suggest a therapeutic advantage (and no discernable toxicity) to combining TRT and ICIs in mice with well-established B16F10 melanomas. We believe that systemically delivered TRT may have advantages over external-beam irradiation in treating disseminated disease, leading to improved overall survival in patients with metastatic melanoma. However, further preclinical studies are warranted to determine the optimum dose regimen (single vs. multiple doses of TRT) and the timing of the 2 therapies.

\section{DISCLOSURE}

This work was funded by National Institute of Health grants R01 CA204018 
(Carolyn Anderson) and P50 CA121973 (Skin and Melanoma SPORE, John Kirkwood, principal investigator) and used the UPMC Hillman Cancer Center Animal Facility, In Vivo Imaging Facility, and Biostatistics Facility (NCI P30 CA047904). No other potential conflict of interest relevant to this article was reported.

\section{ACKNOWLEDGMENTS}

We acknowledge Kathryn Day and Joseph Latoche for assistance with preclinical PET/CT imaging, Solomon Johnson for apoptosis data analysis, Jessie Nedrow for assistance with the initial therapy studies, and John Kirkwood for helpful discussions.

\section{REFERENCES}

1. Saladi RN, Persaud AN. The causes of skin cancer: a comprehensive review. Drugs Today (Barc). 2005;41:37-53.

2. Patel JK, Didolkar MS, Pickren JW, Moore RH. Metastatic pattern of malignant melanoma: a study of 216 autopsy cases. Am J Surg. 1978;135:807-810.

3. Ascierto ML, Melero I, Ascierto PA. Melanoma: from incurable beast to a curable bet-the success of immunotherapy. Front Oncol. 2015;5:152.

4. Melero I, Rouzaut A, Motz GT, Coukos G. T-cell and NK-cell infiltration into solid tumors: a key limiting factor for efficacious cancer immunotherapy. Cancer Discov. 2014;4:522-526.

5. Twyman-Saint Victor C, Rech AJ, Maity A, et al. Radiation and dual checkpoint blockade activate non-redundant immune mechanisms in cancer. Nature. 2015;520: 373-377.

6. Vanneman M, Dranoff G. Combining immunotherapy and targeted therapies in cancer treatment. Nat Rev Cancer. 2012;12:237-251.

7. Dijkgraaf I, Boerman OC, Oyen WJ, Corstens FH, Gotthardt M. Development and application of peptide-based radiopharmaceuticals. Anticancer Agents Med Chem. 2007;7:543-551.

8. Beck-Sickinger AG, Khan IU. Targeted tumor diagnosis and therapy with peptide hormones as radiopharmaceuticals. Anticancer Agents Med Chem. 2008;8: $186-199$.

9. Hyun Y-M, Chung H-L, McGrath JL, Waugh RE, Kim M. Activated integrin VLA-4 localizes to the lamellipodia and mediates T cell migration on VCAM-1. J Immunol. 2009;183:359-369.

10. Kenyon NJ, Liu R, O’Roark EM, Huang W, Peng L, Lam KS. An $\alpha 4 \beta 1$ integrin antagonist decreases airway inflammation in ovalbumin-exposed mice. Eur $J$ Pharmacol. 2009;603:138-146.

11. Ley K, Rivera-Nieves J, Sandborn WJ, Shattil S. Integrin-based therapeutics: biological basis, clinical use and new drugs. Nat Rev Drug Discov. 2016;15:173183.

12. Peng L, Liu R, Marik J, Wang X, Takada Y, Lam KS. Combinatorial chemistry identifies high-affinity peptidomimetics against $\alpha_{4} \beta_{1}$ integrin for in vivo tumor imaging. Nat Chem Biol. 2006;2:381-389.

13. Beaino W, Nedrow JR, Anderson CJ. Evaluation of ${ }^{68} \mathrm{Ga}$-and ${ }^{177}$ Lu-DOTAPEG4-LLP2A for VLA-4-targeted PET imaging and treatment of metastatic melanoma. Mol Pharm. 2015;12:1929-1938.

14. Desgrosellier JS, Cheresh DA. Integrins in cancer: biological implications and therapeutic opportunities. Nat Rev Cancer. 2010;10:9-22.

15. Mattila JT, Beaino W, Maiello P, et al. Positron emission tomography imaging of macaques with tuberculosis identifies temporal changes in granuloma glucose metabolism and integrin $\alpha 4 \beta 1$-expressing immune cells. J Immunol. 2017;199: 806-815.

16. Kirschner AS, Ice RD, Beierwaltes WH. Radiation dosimetry of ${ }^{131}$ I-19-iodocholesterol: the pitfalls of using tissue concentration data [reply]. J Nucl Med. $1975 ; 16: 248-249$.

17. Lewis JS, Wang M, Laforest R, et al. Toxicity and dosimetry of ${ }^{177}$ Lu-DOTAY3-octreotate in a rat model. Int J Cancer. 2001;94:873-877.

18. Balch CM, Buzaid AC, Soong S-J, et al. Final version of the American Joint Committee on Cancer staging system for cutaneous melanoma. J Clin Oncol. 2001;19:3635-3648.
19. Grimaldi AM, Marincola FM, Ascierto PA. Single versus combination immunotherapy drug treatment in melanoma. Expert Opin Biol Ther. 2016;16:433-441.

20. Ma W, Gilligan BM, Yuan J, Li T. Current status and perspectives in translational biomarker research for PD-1/PD-L1 immune checkpoint blockade therapy. $J$ Hematol Oncol. 2016;9:47.

21. Kyi C, Postow MA. Checkpoint blocking antibodies in cancer immunotherapy. FEBS Lett. 2014;588:368-376.

22. Dovedi SJ, Adlard AL, Lipowska-Bhalla G, et al. Acquired resistance to fractionated radiotherapy can be overcome by concurrent PD-L1 blockade. Cancer Res. 2014;74:5458-5468.

23. Deng L, Liang H, Burnette B, et al. Irradiation and anti-PD-L1 treatment synergistically promote antitumor immunity in mice. J Clin Invest. 2014;124:687.

24. Azad A, Lim SY, D'Costa Z, et al. PD-L1 blockade enhances response of pancreatic ductal adenocarcinoma to radiotherapy. EMBO Mol Med. 2017;9: $167-180$.

25. Jeong H, Bok S, Hong B-J, Choi H-S, Ahn G. Radiation-induced immune responses: mechanisms and therapeutic perspectives. Blood Res. 2016;51:157-163.

26. Schaue D, Ratikan JA, Iwamoto KS, McBride WH. Maximizing tumor immunity with fractionated radiation. Int J Radiat Oncol Biol Phys. 2012;83:1306-1310.

27. Kikuchi M, Clump DA, Srivastava RM, et al. Preclinical immunoPET/CT imaging using $\mathrm{Zr}$-89-labeled anti-PD-L1 monoclonal antibody for assessing radiation-induced PD-L1 upregulation in head and neck cancer and melanoma. Oncoimmunology. 2017;6:e1329071.

28. Parker C, Nilsson S, Heinrich D, et al. Alpha emitter radium-223 and survival in metastatic prostate cancer. N Engl J Med. 2013;369:213-223.

29. Heck MM, Retz M, D’Alessandria C, et al. Systemic radioligand therapy with ${ }^{177} \mathrm{Lu}$ labeled prostate specific membrane antigen ligand for imaging and therapy in patients with metastatic castration resistant prostate cancer. J Urol. 2016;196: 382-391.

30. Kratochwil C, Bruchertseifer F, Giesel FL, et al. ${ }^{225}$ Ac-PSMA-617 for PSMAtargeted $\alpha$-radiation therapy of metastatic castration-resistant prostate cancer. J Nucl Med. 2016;57:1941-1944.

31. Miao Y, Shelton T, Quinn TP. Therapeutic efficacy of a ${ }^{177} \mathrm{Lu}$-labeled DOTA conjugated alpha-melanocyte-stimulating hormone peptide in a murine melanoma-bearing mouse model. Cancer Biother Radiopharm. 2007;22:333-341.

32. Schadendorf D, Heidel J, Gawlik C, Suter L, Czarnetzki BM. Association with clinical outcome of expression of VLA-4 in primary cutaneous malignant melanoma as well as P-selectin and E-selectin on intratumoral vessels. J Natl Cancer Inst. 1995;87:366-371.

33. García-Martín AB, Zwicky P, Gruber T, et al. VLA-4 mediated adhesion of melanoma cells on the blood-brain barrier is the critical cue for melanoma cell intercalation and barrier disruption. J Cereb Blood Flow Metab. May 15, 2018 [Epub ahead of print].

34. Moretti S, Martini L, Berti E, Pinzi C, Giannotti B. Adhesion molecule profile and malignancy of melanocytic lesions. Melanoma Res. 1993;3:235-239.

35. Schadendorf D, Gawlik C, Haney U, Ostmeier H, Suter L, Czarnetzki BM. Tumour progression and metastatic behaviour in vivo correlates with integrin expression on melanocytic tumours. J Pathol. 1993;170:429-434.

36. Mittal D, Gubin MM, Schreiber RD, Smyth MJ. New insights into cancer immunoediting and its three component phases: elimination, equilibrium and escape. Curr Opin Immunol. 2014;27:16-25.

37. Zamarron BF, Chen W. Dual roles of immune cells and their factors in cancer development and progression. Int J Biol Sci. 2011;7:651-658.

38. Bui JD, Schreiber RD. Cancer immunosurveillance, immunoediting and inflammation: independent or interdependent processes? Curr Opin Immunol. 2007;19: 203-208.

39. Dunn GP, Koebel CM, Schreiber RD. Interferons, immunity and cancer immunoediting. Nat Rev Immunol. 2006;6:836-848.

40. Paolino M, Choidas A, Wallner S, et al. The E3 ligase Cbl-b and TAM receptors regulate cancer metastasis via natural killer cells. Nature. 2014;507:508-512.

41. Yaguchi T, Sumimoto H, Kudo-Saito C, et al. The mechanisms of cancer immunoescape and development of overcoming strategies. Int J Hematol. 2011;93: 294-300.

42. Schlesinger M, Bendas G. Contribution of very late antigen-4 (VLA-4) integrin to cancer progression and metastasis. Cancer Metastasis Rev. 2015;34:575-591.

43. Laurent J, Speiser DE, Appay V, et al. Impact of 3 different short-term chemotherapy regimens on lymphocyte-depletion and reconstitution in melanoma patients. J Immunother. 2010;33:723-734. 